\title{
La utopía de la implementación del B.I.M en la industria de la construcción en Colombia.
}

\author{
The utopia of the implementation of B.IM in the construction industry in Colombia.
}

\author{
> Edgar Alonso Meneses Bedoya \\ Universidad Nacional de Colombia, Colombia. \\ eamenese@unal.edu.co
}

\author{
> Benjamin Montoya Jaramillo \\ Universidad Nacional de Colombia, Colombia. \\ bmontoya@unal.edu.co
}

\begin{abstract}
The aim of the research is analyze from different case studies, how does implemented BIM in the construction industry in Colombia, benefits, difficulties and drawbacks, trying to answer the questions that arise from the experience of this implementation: how does impact the BIM the industry construction, in a context where technological processes and craft activities with very low operational control converge? and How does this implementation affects companies should incorporate innovative models that require profound organizational changes?.
\end{abstract}

Modelado; Virtual; Construcción; Gestión; infografía

\section{Introducción}

Problemas asociados a la imprecisión de la documentación técnica y a procesos constructivos que avanzan sin la validación de la información por parte de los profesionales encargados de los diseños, entre otros, generan cambios en la ejecución de los proyectos que afectan considerablemente los presupuestos. Esta situación le sirve a Phil Bernstein (Bernstein, 2004) para introducir la principal problemática que se pretende superar con la implementación del B.I.M. en la industria de la construcción: La pérdida de los recursos invertidos asociada a factores de gestión de la información constructiva.

A principios de los años 90 surgen una serie de programas informáticos que posibilitan la construcción virtual de edificaciones, integrando toda la información producida por los diferentes profesionales que intervienen en el diseño en un solo archivo gráfico (modelo virtual), dando inicio con esto al concepto del B.I.M.. Después de mas de 15 años de implementación, las empresas productoras de software y consultoras para su implementación, se dan cuenta que el B.I.M. es más que tecnologías informáticas para la representación e implica una nueva manera de organización para la industria de la construcción, tal como lo presenta Randy Deutsch de la A.I.A. en esta definición: "B.I.M. es la combinación de tecnologías, personas y procesos que apoyan el desarrollo y el intercambio de información para la construcción de edificios y su operación." (Deutsch, 2011). Aquí se destaca que la problemática que propone solucionar el B.I.M., no es exclusivamente tecnológica, sino de gestión de la información, de interrelación multidisciplinar y de estructura organizacional en las empresas constructoras.

\section{Metodología}

La investigación se construye a partir de la experiencia de la implementación de tres proyectos asumidos como casos de Estudio

Proyecto 1: Proyecto de Oficinas en la ciudad de Medellín. Dos Torres, 6 Sótanos de parqueo, 22 Pisos de Oficinas en cada Torre. Total construido 56.000 $\mathrm{Mts}^{2}$. Consorcio de dos empresas constructoras. Tiempo estimado inicial 4 meses. Tiempo real de ejecución 20 meses. Figura 1.

Proyecto 2: Centro Comercial en la periferia de la Ciudad de Medellín, Plataforma de cuatro niveles con dos torres de Oficinas de Ocho Pisos, Total construido 160.000 $\mathrm{Mts}^{2}$. Consorcio de tres empresas constructoras. Tiempo estimado inicial 5 meses. Tiempo real de ejecución 24 meses.

Proyecto 3: Proyecto de vivienda en la periferia de la ciudad de Bogotá, cinco torres de 16 pisos, 320 viviendas. Total construido 23.600 $\mathrm{Mts}^{2}$. Una empresa constructora. Tiempo estimado inicial 3 meses. Tiempo real de ejecución 6 meses.

Para cada uno de los proyectos se realizó un contrato de consultoría cuyo objetivo era realizar el modelado completo del edificio con sus respectivas redes (Modelo BIM) y encontrar las interferencias entres sistemas para prever con antelación los problemas que se pudiesen presentar durante la ejecución de la obra. Figura 2.

Para los proyectos 1 y 2 se esperaba que la empresa constructora entregara la planimetría arquitectónica, estructural y de redes definitivas, lo cual no se cumplió y el modelo debió ser actualizado en numerosas oportunidades. 


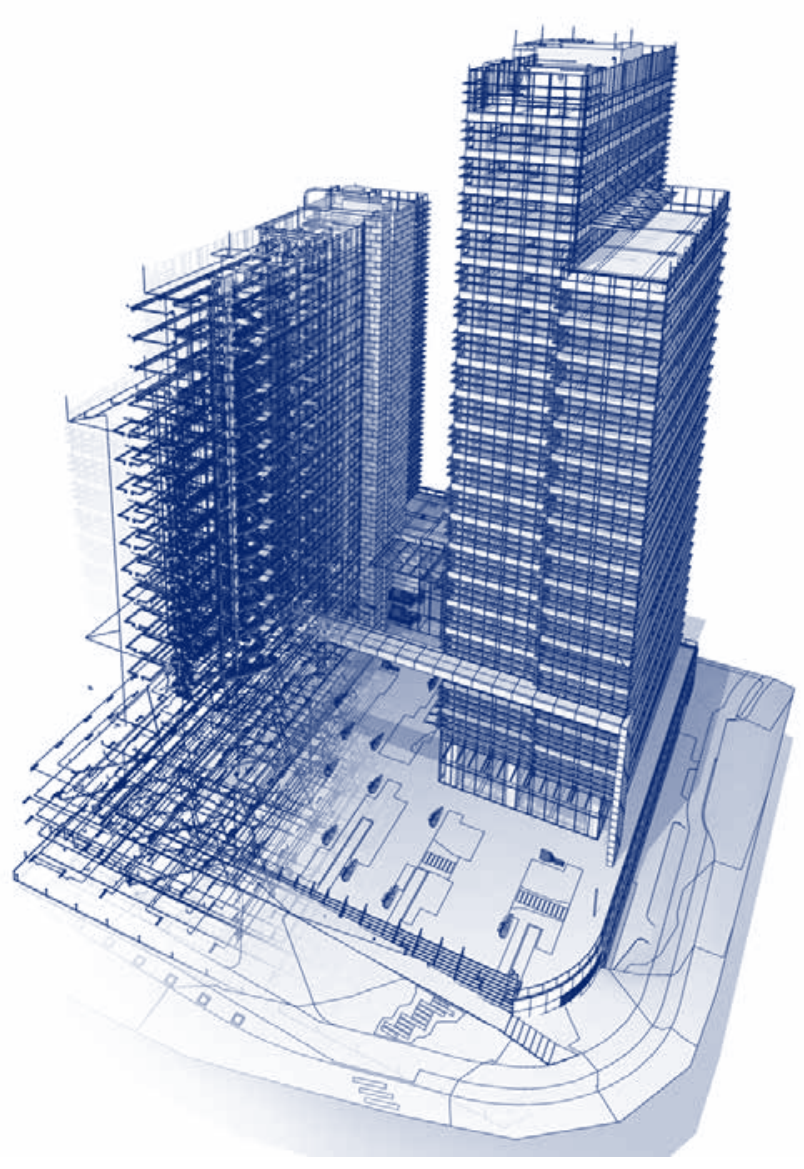

Figura 1. Vista aérea del Proyecto 1. Creado por los autores.

Por otra parte, al analizar la incipiente planimetría de redes suministrada se encontró que ésta era esquemática y no se contaba con suficiente información para elaborar adecuadamente el modelo, razón por la cual se requerían consultas permanentes a los diseñadores, que implicaban rediseńos constantes a estas redes y actualizaciones en el modelo.

En el caso de la planimetría arquitectónica, la situación que se debió afrontar fueron los rediseños constantes que generaban versiones diferentes, e implicaba igualmente actualizaciones permanentes al modelo BIM. En el caso del proyecto 1, se inició el modelado con planos versión 9 y se finalizó el modelo con planos versión 13 .

Haber abordado el proceso de modelado con una información planimetría tan esquemática llevo a que desde los inicios del modelo se encontraron numerosas inconsistencias e interferencias entres sistemas, lo cual hubiese sido problemático durante la ejecución real de la obra. Esta situación evidenció desde el inicio la importancia de implementar esta tecnología, ya que cada diseñador proponía su red por el punto ideal "el centro del pasillo", causando colisiones más que evidentes entre todas las redes, las cuales no habían sido analizadas ni previstas por los diseñadores y residentes de obra.

En la construcción tradicional el residente de obra asume esta falta de información y toma directamente las decisiones sin consultar a los diseñadores. Con el modelo de implementación BIM para

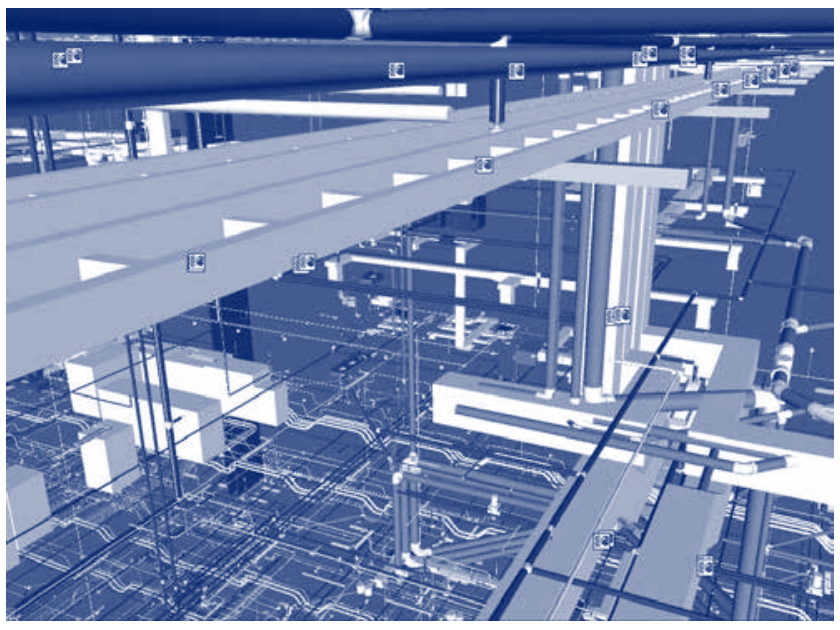

Figura 2. Vista interna de redes Proyecto 1. Creada por los autores

estos proyectos, el encargado de obra sigue asumiendo este papel, pero de una manera "virtual", dentro del modelo BIM, y solo realiza consulta en los casos donde se vea implicada una normativa. Si bien no es conveniente la simultaneidad entre la construcción y el modelado BIM, con esto se evidencia que el uso del modelo para tomar decisiones durante la construcción contribuye sustancialmente a la minimización de errores.

Otra de las problemáticas en la implementación del modelo BIM en estos estudios de caso, se generó debido a que en los proyectos 1 y 2 ya existían avances significativos de la obra cuando se inició el modelado BIM, debido fundamentalmente a que las empresas constructoras tardíamente se percataron de la necesidad de implementar el BIM para minimizar perdidas en la ejecución de la obra.

En el proyecto 3, todos los diseños se entregaron oportunamente y se terminó el modelo sin haber iniciado obras, sin embargo los diseńos de la torre dos a la cinco debieron ser modificados en su totalidad por problemas de mercadeo, lo cual hizo terminar tempranamente el proceso de implementación BIM en estas torres.

Implicaciones Sociológicas de la Implementación B.I.M. Nuestros hallazgos nos han llevado a plantear que más allá de los aspectos tecnológicos, que vienen solucionando las empresas de software y hardware, son los aspectos sociológicos los que deben transformarse para lograr que la construcción pueda incorporar adecuadamente estas tecnologías, ya que mientras estas transformaciones se dan, observamos como son casi infructuosos los esfuerzos que realizan algunas empresas para generar un modelo B.I.M., el cual le permita por lo menos tener el control de la integración de todos los sistemas que configuran el proyecto.

En los casos de estudio presentados, se destacan cuatro interrelaciones entre los actores que participan en la implementación:

1. La empresa contratante y la empresa consultora.

La alta gerencia de las empresas constructoras en algunos casos no visiona la implementación de estas tecnologías como una 
oportunidad de mejora y eficiencia, sino como un ítem más de gastos en el proceso constructivo.

La consultoría contratada con las diferentes empresas involucradas en estos proyectos, requirió grandes esfuerzos de negociación, en el cual el principal problema enfrentado era la percepción de los gerentes de "estar gastando dinero" en un proceso donde no se evidencia el retorno de la inversión. Esta condición hace muy difícil lograr la implementación de estas tecnologías, pues son estos actores los que toman las decisiones estratégicas en las empresas y los que definen las pautas de crecimiento y posicionamiento de las empresas en el mercado.

Por su parte, la empresa consultora ofrece unas expectativas frente a la implementación del modelo, pero debe enfrentar todas las múltiples dificultades que se presentan para garantizar el cumplimiento de los objetivos contractuales. Esto pone a la empresa consultora en una situación de justificación permanente de cada una de las actividades, a fin de que la gerencia quede convencida de que el "gran esfuerzo económico" se justifica.

\section{La empresa consultora y los diseñadores de redes}

La interacción entre la empresa consultora y estos profesionales se establece en diferentes etapas del proceso de implementación y con diferentes niveles de responsabilidad en la toma de decisiones. La principal dificultad encontrada se presenta en el compromiso que implica para los diseñadores asumir el nivel de definición del proyecto según la disciplina comprometida.

En el caso de los arquitectos, están habituados a gestionar la aprobación de un determinado proyecto con una planimetría que se encuentra a nivel de anteproyecto o son "esquemas bien dibujados”. Éstos van definiendo la mayoría de los detalles constructivos durante el mismo proceso de construcción del proyecto y muchas veces dejan esta responsabilidad al constructor que ejecuta la obra. Así mismo, permanentemente están realizando cambios en el proyecto, ajustándolo a los requerimientos del cliente, las decisiones de la gerencia del proyecto o según condiciones encontradas durante la ejecución.

Por su parte los ingenieros eléctricos, hidráulicos y todos aquellos responsables de las redes de sistemas que garantizan la usabilidad de los espacios, están habituados a entregar esquemas de ramificaciones de estos sistemas sobre la planimetría del proyecto, sin confrontar las condiciones reales de alturas libres, de la estructura, del cruce con los demás sistemas, etc. Esta situación es la más problemática, ya que modelar un proyecto M.E.P. (Mechanical, Electrical, Plumbing) con esquemas que a su vez se des actualizan permanentemente a medida que los arquitectos modifican los proyectos, hace absolutamente imposible lograr una adecuada integración y sincronización de las actividades.

Al exigirle a estos profesionales un mayor detalle de los diseños, la relación con el equipo de modelado se hace tensa, ya que es un actor que exige mayor nivel de información de la que el mismo constructor requiere y exige, y para estos profesionales puede significar perdida de dinero en la medida en que los costos iniciales de los diseños no contemplaban este nivel de detalle requerido para el modelo BIM.
3. El supervisor de obra y la empresa consultora.

El equipo responsable de la ejecución del proyecto, son los actores más convencidos de la importancia del uso de estas tecnologías, pues reconocen el nivel de improvisación que se presenta durante la ejecución convencional del proyecto, producto de la falta de documentación detallada de cada uno de los sistemas y su integración.

La interacción con estos actores es la que ha permitido evidenciar el gran aporte del modelado BIM en la construcción, pues ha develado las jerarquías que se asumen en la toma de decisión durante el proceso de construcción y las responsabilidades de todos los profesionales que intervienen. Es importante anotar que un sistema ideal de implementación BIM de un proyecto, la interacción que se daría entre los responsables de la ejecución del proyecto y los responsables del modelado BIM seria mínima, ya que la interacción se hace a través del modelo y éste debe garantizar que la ejecución se pueda hacer de manera eficiente contando con una documentación completamente validada.

4. El supervisor de obra y los diseñadores de redes.

El proceso implementado genero una interacción mayor entre el supervisor y los diseñadores, ya que éste comenzó a exigirle a los diseńadores, lo que en un proceso constructivo convencional, solucionaba en sitio: desplazamiento de redes, fijación de alturas, pases por elementos estructurales, entre otros. El flujo de información requerida para el modelado del proyecto y por ende para la construcción, depende de la actitud asumida por los diseñadores, que no en todos los casos están disponibles y predispuestos a atender los requerimientos.

Otro aspecto que se desprende de estas interrelaciones, se fundamenta en la afirmación de Bernstein "La evolución de la aplicación del BIM viene en paralelo con la voluntad de colaborar y compartir información del proyecto hacia una práctica integrada de la cual se habla mucho en la industria" (Bernstein, 2006). En esta se evidencia que para la implementación del BIM hace falta más voluntad que talento.

\section{Resultados}

Proponemos los siguientes modelos de implementación como instrumentos que guían la forma como debe concebirse el desarrollo de un proyecto BIM en el contexto local.

Modelo ideal: Interpretado de las propuestas de autores tales como Phil Bernstein (Bernstein, 2012). En éste cada uno de los profesionales que intervienen en el proceso trabaja de manera colaborativa en un mismo modelo digital que contiene toda la información del proyecto. Los procesos de actualización y cambios son informados en tiempo real a cada uno de los profesionales encargados de los sistemas del proyecto, quienes tendrán la responsabilidad de actualizar de inmediato su propio sistema en el modelo centralizado. En este caso el modelo se construye desde las etapas iniciales de diseńo y va ganando en complejidad en la medida en que el proyecto adquiere mayor definición para su construcción.

Este modelo de implementación es aún impensable en nuestro entorno, ya que son pocos los profesionales que desde su propia 
área disciplinar han incorporado estas tecnologías y metodologías de trabajo, y así mismo son muy pocas las empresas que cuentan con personal calificado en cada una de las áreas, que garantice poder avanzar en el desarrollo de un proyecto con una perspectiva BIM desde los inicios del proyecto.

Modelo implementado: Consiste en contratar el modelado BIM del proyecto virtualmente con el fin de analizar la confiabilidad de la documentación planimetría del proyecto y evaluar los posibles casos de falta de coordinación entre sistemas que generan problemas durante la ejecución del proyecto. Esto técnicamente se denomina análisis de interferencias mediante el modelo BIM. Generalmente este modelo se basa en una relación contractual con una empresa consultora externa, que debe asumir las problemáticas asociadas al difícil flujo de la información.

La gran dificultad de este modelo de implementación BIM es no contar con la documentación final y detallada de cada uno de los sistemas del proyecto y enfrentarse a un flujo de trabajo en el cual la ejecución del proyecto avanza en paralelo a la creación del modelo digital. Adicionalmente este tipo de modelo es poco flexible frente a los cambios en los diseńos que se puedan llegar a presentar durante el modelado, ya que cualquier cambio en un detalle o variación de un sistema puede implicar una actualización del modelo que genera atrasos en las programaciones de ejecución.

Modelo alternativo: surge metodológicamente de las dificultades encontradas en los casos de estudio. Éste propone un estadio intermedio entre el modelo ideal y el implementado, ya que reconoce que las empresas no disponen actualmente del personal idóneo para el desarrollo del modelado BIM y a su vez se presentan problemas en el flujo de la información cuando se contrata una empresa externa.

Este modelo propone que un proveedor de servicios tecnológicos especializados (empresa consultora) ponga a disposición de la empresa constructora un personal altamente capacitado y con los recursos necesarios (equipos y software licenciado), puesto directamente en la empresa constructora y bajo la supervisión de un manager BIM, con el fin de que se pueda hacer un acompańamiento directo al desarrollo del proyecto, incluso desde los mimos inicios del diseño. De esta manera se mejora el flujo de la información, ya que se establece una relación más directa entre los integrantes el equipo de trabajo y se evitan demoras en los tiempos de respuesta de los profesionales de las diferentes disciplinas que intervienen en el proyecto y los cambios durante el proceso se afrontan de una manera más eficiente.

Este acompañamiento debe estar presente durante las etapas de diseño y ejecución de la obra, ya que en la etapa de ejecución el modelo BIM es un instrumento de control.

\section{Conclusiones}

La investigación pone en evidencia que "Construir Virtualmente" un modelo B.I.M. es casi tan importante como la misma ejecución, pues otorga mayor control sobre el proyecto y reduce los umbrales de incertidumbre en la ejecución.

El modelo BIM debe garantizar que el proyecto pueda ser modificable de manera eficiente durante los proceso de diseńo ajustándose a cada etapa de definición del proyecto. Si el Modelo
BIM se constituye en un elemento rígido que condiciona la evolución del proyecto, esto se convierte en un factor desestimulante para la implementación de estas tecnologías, ya que ninguna empresa podría admitir que un proceso de construcción virtual de una edificación condicione la evolución de un proyecto para justarlo a unas realidades cambiantes en el proceso de diseño. Por esto es fundamental que la empresa aborde el proceso de creación del modelo BIM desde los orígenes del proyecto, ya que esto le permitirá conocer con precisión las implicaciones de cualquier tipo de cambio que se quiera hacer al proyecto y podrá proceder a actualizarlo según sus propias necesidades.

Un flujo de trabajo con una empresa externa que modele el proyecto BIM se hace inviable en la medida en que el proyecto sufre muchas modificaciones durante el proceso de modelado. Esto se evidencia en los dos casos de estudio mencionados y fue el factor más complejo de manejar.

En las experiencias presentadas la característica predominante fue el abordar un ejercicio de modelado geométrico de los sistemas constitutivos del proyecto sin información detallada y sin una respuesta oportuna por pate de los responsables del diseño para facilitar la elaboración del modelo. Ante una falta de respuesta, la alternativa propuesta por los responsables de la ejecución de la obra se constituían en la solución que finalmente se detallaba en el modelo, esto sin contar muchas veces con el aval del responsable del diseño. Estos ajustes a los diseños no quedan documentados en la planimetría base, lo cual significa que se pierde la memoria de las decisiones tomadas en la obra y finalmente el proyecto queda con una planimetría desactualizada que puede significar problemáticas para el control de la operación de la edificación durante su vida útil en contrasentido de la filosofía BIM. Debería ser una exigencia normativa que se entregaran planos actualizados con todos los ajustes realizados al proyecto durante la etapa constructiva y que debe contar con el visto bueno de cada disciplina involucrada en los procesos de ajuste.

Para cualquier modelo de implementación debe fijarse como meta que en la etapa de diseńo, el modelo BIM y el análisis de interferencias debe estar culminado en su totalidad antes del inicio de la obra. Debido a la complejidad de las interrelaciones entre sistemas es inviable pensar en abordar por etapas el modelado BIM e ir incorporando los sistemas en función al avance de la obra. Esta condición completamente normal en los modelos de programación de obra física, no funciona para el objetivo del modelo BIM, ya que justamente se busca encontrar problemas asociados a la integración de sistemas que han sido pensados y diseñados por diferentes profesionales, muchas veces sin lograr una adecuada coordinación e integración.

La construcción de un modelo BIM minimiza el nivel de improvisación que se genera por la falta de coordinación entre la documentación planimetría de cada uno de los sistemas. Una ejecución coordinada a partir de una planimetría extraída de un modelo BIM puede disminuir ostensiblemente el nivel de error en la ejecución, por esto es fundamental actualizar la planimetría después de la construcción del modelo BIM.

El modelo BIM puede llevarse directamente a la obra a través de dispositivos móviles y plataformas tecnológicas que permiten 
acceder a la información virtual. Esto ayudaría enormemente en el proceso de ejecución, ya que el modelo tridimensional sustituye con información veraz que elimina la ambigüedad de la posible interpretación de un plano, indicando como es que se espera que se ejecute un determinado componente o sistema. Esta eliminación de la ambigüedad se constituye en uno de los elementos más potentes de la simulación del proyecto mediante el modelo BIM y debe existir como recurso para la toma de decisiones en la obra.

Mientras la industria de la construcción siga admitiendo la ejecución de proyectos a partir de información esquemática que varía durante el mismo proceso de ejecución, será prácticamente imposible tener el control del proyecto a partir del modelo BIM. Por esto urge la necesidad de normatizar las condiciones que debe cumplir cada disciplina para garantizar un estándar de calidad óptimo con la suficiente información para la ejecución de un proyecto.

La implementación de estas tecnologías debe ser un proceso gradual en el cual todos los actores adquieran paulatinamente la "cultura" de la documentación exhaustiva del proyecto antes de la ejecución, pues cuando se aborda como un proceso impositivo sin el convencimiento necesario, cada actor se puede convertir en un elemento adverso que llevará al fracaso cualquier esfuerzo.

El trabajo colaborativo es esencial para cualquier implementación, pues en la medida en que la información fluya, sea compartida y atendida a tiempo, podrá garantizarse la eficiencia en la interacción. Comprometer todo el equipo de trabajo en la "filosofía" BIM, mientras no estén convencidos no dedicarán su mejor esfuerzo al proceso.

Es necesario abandonar la idea del proceso constructivo en fases, que tiende a crear una interacción lineal donde cada miembro del equipo actúa en la "fase" que le corresponde, y adoptar un modelo donde convergen las diferentes fases en paralelo interactuando constantemente entre ellas.

\section{Bibliografía}

Bernstein, P.G. \& Pittman, J.H. (2004). Barriers to the adoption of building information modeling in the building industry, Autodesk building solutions.

Bernstein P.G. (2006). BIM Adoption: Finding Patterns for a New Paradigm. Tomado de http://www.di.net/articles/bim_adoption_finding_patterns_for/

Bernstein, P. G. \& Deamer, P. (2010). Building (in) the Future: Recasting Labor in Architecture. New York: Princeton Architectural Press.

Deutsch, R. (2011). B.I.M. and Integrated Design: Strategies for Architectural Practice. Hoboken, New Jersey: John Wiley \& Sons, Inc 